\title{
Monte Carlo simulations for scattering of electromagnetic waves from perfectly conductive random rough surfaces
}

\author{
M. Nieto-Vesperinas and J. M. Soto-Crespo \\ Instituto de Optica, Consejo Superior de Investigaciones Cientificas, Serrano 121, 28006-Madrid, Spain
}

Received June 8, 1987; accepted July 30, 1987

\begin{abstract}
Numerical calculations of mean scattered intensities by simulation of one-dimensional perfectly conductive random rough surfaces are presented. Results relative to backscattering enhancement and more accurate criteria for the validity of the Kirchhoff approximation are obtained. This method can also be used for assessing perturbative theories and for further experiments.
\end{abstract}

The scattering of light and other electromagnetic waves from rough surfaces has long been a subject of interest. Recently much attention has been focused on this subject in connection with the discovery of the weak localization of photons in random media. This is manifested by an enhancement of intensity in the backscattering direction when multiple scattering takes place. ${ }^{1-4}$ This phenomenon has been observed recently in extremely rough random surfaces with large conductivity. 5,6 However, none of the current reported theories has predicted this effect so far. This may be because multiple-scattering models are perturbative, based either on the Rayleigh hypothesis ${ }^{7,8}$ or on the extinction theorem ${ }^{9-13}$ as formulated in Ref. 14 . (A good review may be found in Ref. 15.) Therefore all calculations so far have been restricted to small roughness, $\sigma \ll \lambda$. A perturbative analysis established in Ref. 16 accounts for the existence of backscattering peaks for small roughness when there is interaction with surface polaritons but does not apply to the observations of Refs. 5 and 6.

The purpose of this Letter is to show that the formulation based on the extinction theorem (which in principle constitutes an exact approach to the problem) can account for the enhanced backscattering phenomenon if one uses it without perturbative series approximations. As a matter of fact, that method permits numerical calculations that may be performed for almost any roughness $\sigma$ and correlation length $T$ of the surface profile within the limitations of today's computers (which require that the surface model be one dimensional). This is important in our opinion, since it permits the prediction of interesting results in quite different situations, including those of large $\sigma$ or large incidence angles, not yet considered in multiple-scattering theories. Further, it can yield an exhaustive collection of scattering data with the possibility of establishing more-accurate criteria of validity of the well-known Kirchhoff approximation (KA). ${ }^{17}$

We present Monte Carlo computer simulations for the scattered intensities from samples of one dimensional (1-D) perfectly conductive random surfaces, $z=$ $D(x)$, with rms $\sigma$, normal statistics, and a Gaussian correlation function $\exp \left(-\tau^{2} / T^{2}\right), \tau=x-x^{\prime}$. The surfaces are generated by following a procedure used in Ref. 18, that is, a sequence of random numbers, with normal statistics, zero mean, and variance unity is constructed from another series of random numbers uniformly distributed in $(0,1)$ directly generated by the computer. Then the former sequence is rescaled to the desired variance, and the result, say, $\left\{y_{k}\right\}$, is correlated with a Gaussian, $(2 / \sqrt{\pi} T)^{1 / 2} \exp \left[-2(k / t)^{2}\right]$, to get the appropriate surface profile $\left\{z_{k}\right\}$ with a Gaussian correlation function.

For a given incident linearly polarized plane wave $\hat{u} \exp \left[i k_{0}\left(x \sin \theta_{0}-z \cos \theta_{0}\right)\right]\left(k_{0}=2 \pi / \lambda, \hat{u}\right.$ is a unit vector specifying the polarization, and $\theta_{0}$ is the incidence angle) upon a certain surface sample, the corresponding electric current $\mathbf{J}(\mathbf{x})$ is determined from the extinction theorem boundary condition, namely, Eqs. (15) and (17) of Ref. 14 for the electric field for $s$ waves and Eqs. (18) and (20) of Ref. 14 for the magnetic field for $p$ waves. (Note that only the electric current term will contribute for perfect conductors. Also, since the surface is $1-D$, no depolarization occurs.) Then the electric current is introduced into the far-zone expression for the scattered field, and the angular distribution of scattered intensity for that sample is obtained. This intensity is normalized to its total area, so that the result is independent of the total incident energy, illuminated sample length, and other constants appearing in the asymptotic far-zone expressions. For each incident wave, the intensities for 200 samples were calculated, and then their average was taken; 220 points were taken in each surface sample to perform the $x$ integrals. Calculations were done on a CDC Cyber 180/855 computer. Small dispersion in the areas of the calculated nonnormalized intensities was considered a criterion of numerical consistency of the results. The statistical bias of the mean intensities produced asymmetric curves for $\theta_{0}=0^{\circ}$, which were artificially symmetrized by averaging every two values at scattering angles $\theta$ and $-\theta$.

For brevity we present only a few representative results. Figs. 1-3 show the angular distribution of mean scattered intensities for $s$ waves (solid lines) and $p$ waves (dotted lines) for $T=0.2 \lambda, \lambda$, and $1.8 \lambda$, respectively, and for different values of $\sigma$, (also in units of $\lambda$ ). 

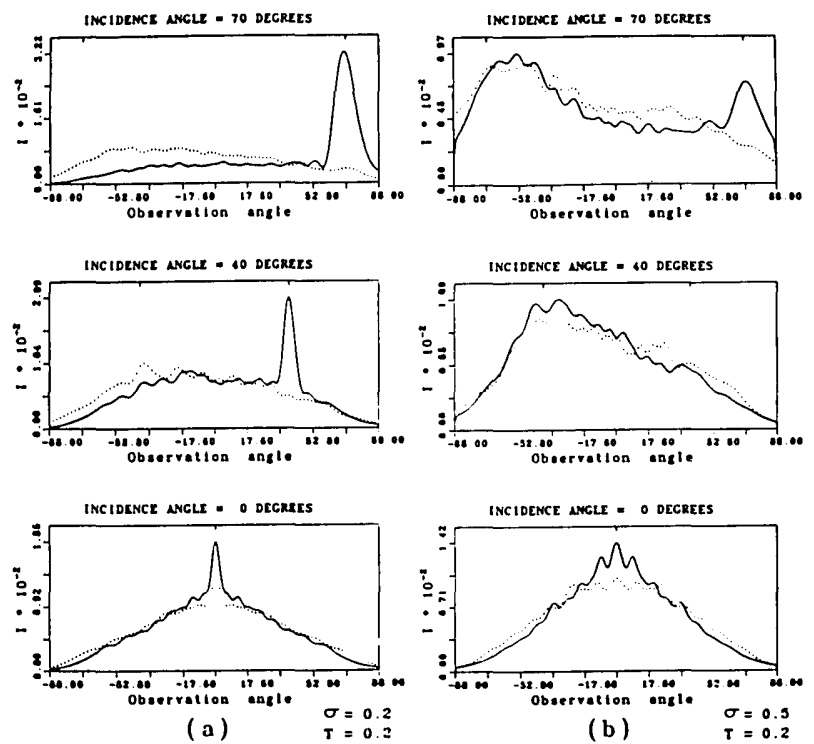

Fig. 1. Mean scattered intensity for (a) $T=0.2 \lambda, \sigma=0.2 \lambda$, (b) $T=0.2 \lambda, \sigma=0.5 \lambda$, and several angles of incidence. Solid curves, $s$ waves; dotted curves, $p$ waves.
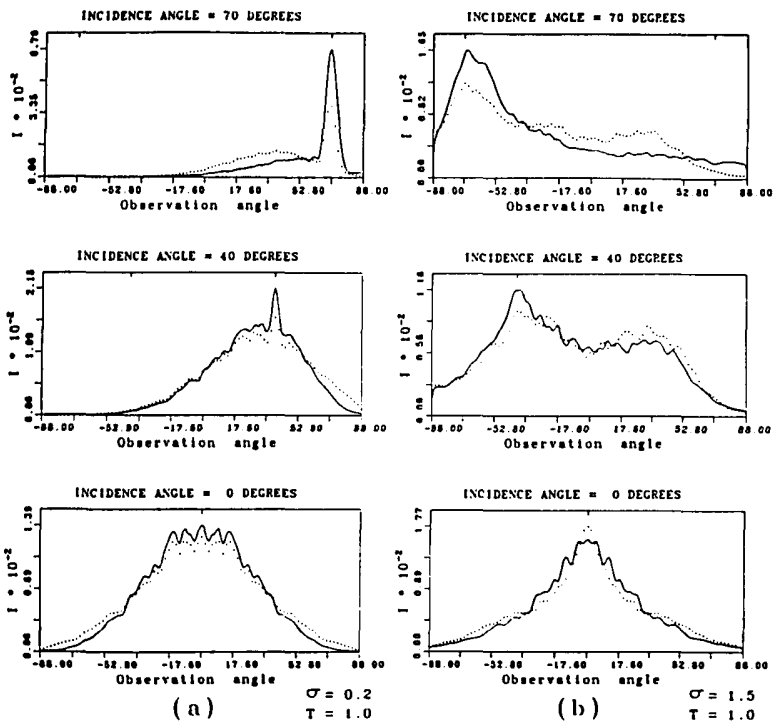

Fig. 2. Same as Fig. 1 for (a) $T=\lambda, \sigma=0.2 \lambda$; (b) $T=\lambda, \sigma=$ $1.5 \lambda$.

It is seen that the specular peaks for both $s$ and $p$ waves are dominant as $\sigma \ll \lambda$ and $T$ increases with respect to $\sigma$ and $\lambda$ [Figs. 2(a) and 3(a)]. (Of course these peaks are larger with respect to the background as $\theta_{0}$ increases.) Conversely, the backscattering effect is dominant as $\sigma$ increases, specifically from values of $\sigma$ similar to or greater than $T$ [Figs. 1(b), 2(b), and 3(b)]. Note that in Figs. 2(a) and 3(a) there is an immersion of the specular peak into the background halo for lower $\theta_{0}$ because of its diffraction width, as the illuminated sample is finite. An interesting phenomenon appears at low T; see Fig. 1(a). There $\sigma$ is comparable with $T$, and thus small $\sigma$ still gives rise to a large specular peak for the $s$ component. However, the $p$ component has a drastically different behavior: it is broadly seattered in all directions, and its intensity distribution is skewed toward the backscattering direction, its backscattering peak being sharper as $\theta_{0}$ decreases. For the same value of $T$ and larger $\sigma$ [Fig. 1(b)], both $s$ and $p$ waves yield a peak in the backscattering, although at larger $\theta_{0}$ the specular peak is still noticeable for $s$ waves. In this connection, a sort of competition between the specular and backscattering directions exists for different values of $T$ and $\sigma$. This can even shift the maximum with respect to either direction [see, e.g., Fig. 3(b) at $\theta_{0}=40^{\circ}$ for $p$ waves]. Figures 2(b) and 3(b) also illustrate some aspects of this. Incidentally, these two figures are qualitatively equivalent to Fig. 2 of Ref. 5, which corresponds to an experiment with $\theta_{0}=20^{\circ}, T=2.2 \lambda$, and $\sigma=1.6 \lambda$.

It was remarked in Ref. 19 that the backscattering peak could not be observed unless ensemble averages were made. This is clearly confirmed in our results. Figure 4(a) shows the scattered intensity for $T=\lambda, \sigma=$ $1.5 \lambda$, and $\theta_{0}=40^{\circ}$ for one surface sample ( $p$ waves above, $s$ waves below). Large speckle fluctuations are exhibited that swamp the backscattering and specular
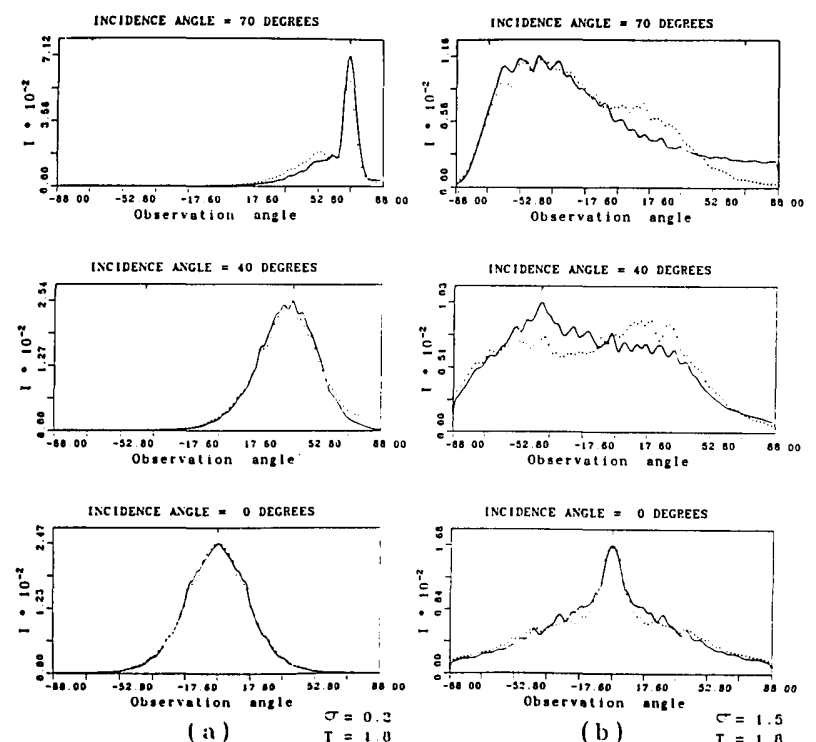

Fig. 3. Same as Fig. 1 for (a) $T=1.8 \lambda, \sigma=0.2 \lambda$; (b) $T=1.8 \lambda$, $\sigma=1.5 \lambda$.
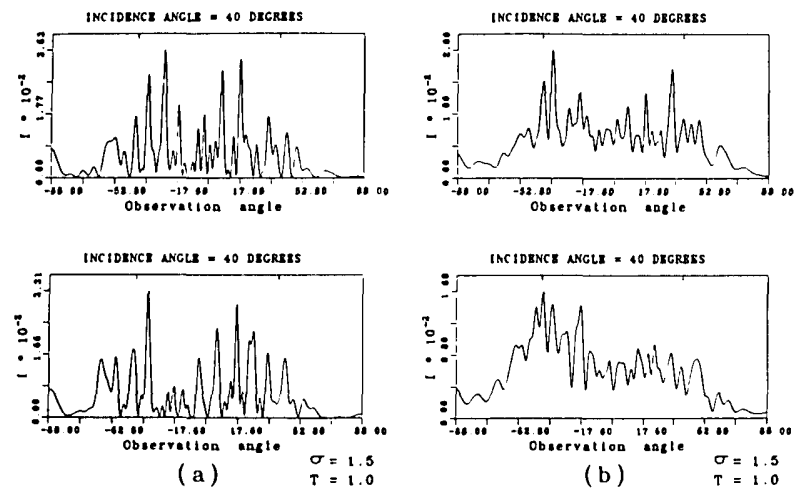

Fig. 4. (a) Scattered intensity from one sample with $T=\lambda$, $\sigma=1.5 \lambda$ for $s$ waves (lower curve) and $p$ waves (upper curve). (b) Mean scattered intensity from ten samples with $T=\lambda, \sigma$ $=1.5 \lambda$ for $s$ waves (lower curve) and $p$ waves (upper curve). 
maxima. Speckle tends to disappear as one starts averaging. Figure $4(\mathrm{~b})$ shows the same scattered intensity as Fig. 4(a) but for averaging over 10 samples. This figure and the average in Fig. 2(b) at $\theta_{0}=40^{\circ}$, which involves 200 samples, confirms the above statement in the case of rough surfaces.

Assessments of the validity of the KA can also be made from these calculations. Figures 2(a) and 3(a) show almost identical distributions for $s$ and $p$ waves for $\theta_{0}$ up to about $40^{\circ}$ in Fig. 2(a) and for any $\theta_{0}$ in Fig. 3(a). We have made calculations with the $\mathrm{KA}$ and obtained identical results (the KA does not account for depolarization). We have obtained an approximate agreement even for $T=0.2 \lambda$ and $\sigma=0.05 \lambda$. If one examines these cases one observes that they all correspond to values $\sigma /(T) \cos \theta_{0}<0.2$, regardiess of the value of $T / \lambda$. We propose to adopt this as a more accurate criterion for the validity of the KA in random surfaces. [It remains to be seen whether the criterion also holds for $\sigma / \lambda$ very large, namely, $\sigma /\left(\lambda \cos \theta_{0}\right) \gtrsim 10$, and for periodic surfaces.] This value is considerably more refined than the one $(T>2 \lambda$ and $\sigma / T<0.05)$ proposed in Ref. 20 and explains the agreement of the KA with actual experiments at $T \simeq \lambda, \sigma \simeq 0.2 \lambda$, and $\theta_{0}$ $=20^{\circ}$ found in Ref. 6 .

Finally, we believe that these calculations may be useful in checking other results, such as the range of validity of perturbative theories, scattering from nonGaussian surfaces, and further experiments.

We thank J. C. Dainty, E. R. Méndez, and K. A. O'Donnell for sharing their experimental data with us. This research was supported by the Comision Asesora de Investigación Científica y Técnica under grant $2502 / 83$. The research of J. M. Soto-Crespo is supported by the Ministerio de Educación y Ciencia.

\section{References}

1. Y. Kuga and A. Ishimaru, J. Opt. Soc. Am. A 1, 831 (1984).

2. L. Tsang and A. Ishimaru, J. Opt. Soc. Am. A 1, 836 (1984).

3. H. P. Van Albada and A. Lagendijk, Phys. Rev. Lett. 55, 2692 (1985).

4. P. E. Wolf and G. Maret, Phys. Rev. Lett. 55, 2696 (1985).

5. E. R. Méndez and K. A. O'Donnell, Opt. Commun. 61, 91 (1987).

6. K. A. O’Donnell and E. R. Méndez, J. Opt. Soc. Am. A 4, 1194 (1987)

7. S. O. Rice, Comm. Pure Appl. Math. 4, 351 (1951).

8. G. R. Valenzuela, IEEE Trans. Antennas Propag. AP15, 552 (1967).

9. F. Toigo, A. Marvin, V. Celli, and N. R. Hill, Phys. Rev. B 15, 2371 (1977).

10. J. Shen and A. Maradudin, Phys. Rev. B 22, 4234 (1980).

11. M. Nieto-Vesperinas, J. Opt. Soc. Am. 72, 539 (1982).

12. D. P. Winbrenner and A. Ishimaru, J. Opt. Soc. Am. A 2, 2285 (1985).

13. D. Maystre and J. P. Rossi, J. Opt. Soc. Am. A 3, 1276 (1986).

14. D. N. Pattanayak and E. Wolf, Opt. Commun. 6, 217 (1972).

15. G. S. Brown, Wave Motion 7, 195 (1985).

16. V. Celli, A. A. Maradudin, A. A. Marvin, and A. R. McGurn, J. Opt. Soc. Am. A 2, 2225 (1985).

17. P. Beckmann and A. Spizzichino, The Scattering of E.M. Waves from Rough Surfaces (Macmillan, New York, 1963).

18. A. K. Fung and M. F. Chen, J. Opt. Soc. Am. A 2, 2274 (1985).

19. S. Etemand, R. A. Thompson, and M. Andrejco, Phys. Rev. Lett. 57, 575 (1986).

20. M. Nieto-Vesperinas and N. Garcia, Opt. Acta 28, 1651 (1981). 